\title{
Genetic cause of immune dysregulation - one gene or two?
}

\author{
Stuart G. Tangye \\ Immunology Division, Garvan Institute of Medical Research, Darlinghurst, New South Wales, Australia. St. Vincent's Clinical School, University of New South Wales, Sydney, New South Wales, Australia.
}

\begin{abstract}
Some autoimmune disorders are monogenetic diseases; however, clinical manifestations among individuals vary, despite the presence of identical mutations in the disease-causing gene. In this issue of the $\mathrm{JCl}$, Massaad and colleagues characterized a seemingly monogenic autoimmune disorder in a family that was linked to homozygous loss-of-function mutations in the gene encoding the endonuclease Nei endonuclease VIII-like 3 (NEIL3), which has not been previously associated with autoimmunity. The identification of an unrelated healthy individual with the same homozygous mutation spurred more in-depth analysis of the data and revealed the presence of a second mutation in a known autoimmune-associated gene. Animals lacking Neil3 had no overt phenotype, but were predisposed to autoantibody production and nephritis following exposure to the TLR3 ligand poly(I:C). Together, these results support further evaluation of the drivers of autoimmunity in supposedly monogenic disorders.
\end{abstract}

\section{Genetics of immune dysfunction}

It is generally recognized that the first primary immunodeficiency was described in 1952, when Ogden Bruton reported a male patient who lacked all serum gammaglobulins (antibodies/immunoglobulins) and suffered from recurrent bacterial infections (1). Remarkably, regular subcutaneous infusion of concentrated human immune serum globulins protected the patient from sepsis (1), demonstrating a link between the absence of gammaglobulin and severe bacterial infections and the possible feasibility of treating such individuals with gammaglobulin replacement therapy. Forty years later, the gene responsible for Bruton's or X-linked agammaglobulinemia was identified as BTK (Bruton's tyrosine kinase) $(2,3)$. Today, with the incredible advances that have been made in next-generation and high-throughout sequencing technologies over the last decade, mutations in more than 300 genes have been discovered that cause primary immunodeficiencies (4). In fact, the clinical manifestations of these conditions would be more appropriately described as immune dysregulation because these conditions often go well beyond susceptibility to infectious diseases and include autoinflammation, autoimmunity, allergic disease, and even malignancy $(4,5)$.

While these diseases are often considered to be Mendelian and monogenic in nature, the clinical presentation of individuals with diseases that result from mutations in the same gene can be extremely diverse, ranging from mild disease to fatal infections or autoimmunity $(4,6)$. This variability is also observed in families with the same genetic defect, indicating that disease manifestation is not simply a genotype/phenotype effect. In fact, some mutations remain clinically silent, as evidenced by asymptomatic carriers of ostensibly pathogenic gene

Related Article: p. 4219

Conflict of interest: The author has declared that no conflict of interest exists.

Reference information: J Clin Invest. 2016;126(11):4065-4067. doi:10.1172/JCI90831.

mutations $(7,8)$. This incomplete penetrance of genetic traits is often attributed to environmental or epigenetic influences that modulate the impact of gene mutations on disease pathogenesis (6). However, another possibility is that the condition is actually digenic or multigenic, inasmuch as a mutation in a second gene is required for full-blown clinical disease. This raises the question as to whether a particular disease is monogenic with incomplete penetrance or multigenic in nature. While GWAS studies certainly suggest that many autoimmune diseases are polygenic (9), this hypothesis has rarely been tested in the setting of conditions such as primary immunodeficiencies that are considered to result from mutations in a single gene.

In the past, it has been challenging to formally test the concept that supposed monogenetic disorders may instead be polygenetic because, typically, few candidate genes would be analyzed. Moreover, any mutation found in one of the analyzed genes was assumed to be the deleterious genetic lesion - a reasonable conclusion. However, whole-exome and genome sequencing have revealed that mutations are common in the human population and the vast majority of these genetic changes are clinically silent (6). While we tend to focus on a short list of single candidate genes that are likely to be pathogenic, we need to consider the possibility that the disease phenotype may result from genetic epistasis. In this issue, Massaad et al. provide a compelling example whereby immune dysregulation and autoimmunity due to mutation in a disease-associated gene is exacerbated by a mutation in a completely unrelated gene (10).

\section{An asymptomatic individual tells the tale}

Massaad et al. describe three siblings from a consanguineous Kuwaiti family suffering from recurrent bacterial and fungal infections, defective peripheral B cell tolerance, 
and severe autoimmunity. This condition was uniformly fatal in the second decade of life. Whole-genome sequencing analysis revealed homozygous mutations in Nei endonuclease VIII-like 3 (NEIL3), which encodes the endonuclease VIII-like enzyme involved in DNA base excision repair, in the three affected individuals. Both parents and all other healthy siblings were heterozygous for this mutation, indicating complete genetic segregation with disease. The specific mutation abolished the enzymatic activity of NEIL3, thereby affecting DNA repair in these individuals and establishing the potential pathogenicity of this genetic lesion. As defects in DNA repair have been associated with human autoimmunity (11), it would not be inappropriate to conclude that deleterious mutations in NEIL3 represent a novel cause of immune dysregulation.

However, Massaad and colleagues also identified the same NEIL3 mutation in a single unrelated healthy adult - a finding that, at face value, disproves the hypothesis that mutations in NEIL3 are disease causing. Despite no presentation of disease, serum from the asymptomatic adult contained high levels of autoantibodies, and $\mathrm{B}$ cells from this subject exhibited defects in peripheral tolerance, attributes that were similar to those documented for the three initial cases (10). The paradoxical finding of a healthy carrier with a putative pathogenic homozygous mutation and elevated yet subclinical autoantibody titers led Massaad et al. to reassess the data from their initial whole gene-sequencing analysis of the index patients. This analysis resulted in the identification of a cryptic duplicated homozygous mutation in LRBA (encoding LPS-responsive and beige-like anchor) that results in loss of LRBA protein expression. Notably, biallelic, null mutations in $L R B A$ have been reported by several groups (including authors of the current study) to cause systemic autoimmunity, splenomegaly, recurrent infections, and hypogammaglobulinemia (12-17). At the cellular level, LRBA deficiency compromises the generation and function of regulatory $\mathrm{T}$ cells, promotes immune cell apoptosis, and reduces autophagy $(12,14-16)$, all of which would contribute to defective immune regulation and progressive autoimmunity. Thus, the three affected siblings characterized by Massaad et al. bore homozygous muta- tions in both $L R B A$, which have clearly been associated with immune dysregulation, and NEIL3, which had not previously been associated with disease. As defects in $B$ cell tolerance in one of the patients harboring mutations in both NEIL 3 and $L R B A$ were no greater than those in individuals with a mutation in NEIL3 or LRBA (10), the relative contribution of these mutations to disease were not clear. Moreover, it needed to be determined whether NEIL3 mutations could promote disease caused by LRBA deficiency.

Consequently, Massaad et al. studied Neil3-deficient mice to evaluate the contribution of this gene to the observed clinical phenotypes. Mice lacking Neil3, not unlike the healthy individual with the single NEIL3 mutation, were largely normal, with intact development and function of all immune cell lineages tested and no obvious autoimmunity. However, again paralleling the healthy asymptomatic carrier, Neil3-deficient mice exhibited substantially increased levels of autoreactive IgM and IgG antibodies. The more striking finding though was that production of autoantibodies, as well as the incidence of autoimmunity, in Neil3-deficient mice was greatly increased following repeated administration of the TLR3 ligand polyinosinic:polycytidylic acid (poly[I:C]), which mimics infection with double-stranded RNA viruses. Furthermore, Neil3-deficient murine $\mathrm{B}$ and $\mathrm{T}$ cells exhibited greater apoptosis following activation in vivo and in vitro. Thus, while not directly causing a break in self-tolerance, NEIL3 deficiency appears to predispose to autoimmunity. In Neil3deficient mice, further insults in the form of pathogen infection may cause frank autoimmunity. In humans, NEIL3 mutations may exacerbate the immune dysregulation caused by $L R B A$ mutations. It is tempting to speculate that some of the variability in the clinical phenotype of LRBA deficiency, including the identification of asymptomatic individuals $(15,17)$, could be attributed to the presence or absence of second-hit mutations, such as those reported by Massaad et al. for NEIL3 (10). A major goal for future studies will be to determine whether specific clinical phenotypes result uniquely from mutations in NEIL3 or LRBA or whether the combination of defects in both genes underlies disease onset, incidence, and severity.

\section{Concluding remarks}

These findings of Massaad et al. are interesting because they illustrate the benign (subclinical) nature of specific gene mutations, yet also reveal potential combinatorial effects of multiple genetic lesions. This result provides a salient lesson about the need to consider the possible consequences of mutations in unanticipated genes that are observed in essentially all studies employing whole genome sequencing as a platform to discover the molecular cause of human diseases (6). Studies such as that of Massaad et al. will pave the way for the elucidation of additional gene mutations that cooperate to result in clinical phenotypes - and offer a glimpse of the complexity of human genetic diseases that may occasionally be oligogenic, rather than monogenic.

Address correspondence to: Stuart Tangye, Immunology Division, Garvan Institute of Medical Research, 384 Victoria St., Darlinghurst, New South Wales 2010, Australia. Phone: 61.2.9295.8455; E-mail: s.tangye@ garvan.org.au.

1. Bruton OC. Agammaglobulinemia. Pediatrics. 1952;9(6):722-728.

2. Rawlings DJ, et al. Mutation of unique region of Bruton's tyrosine kinase in immunodeficient XID mice. Science. 1993;261(5119):358-361.

3. Tsukada S, et al. Deficient expression of a B cell cytoplasmic tyrosine kinase in human X-linked agammaglobulinemia. Cell. 1993;72(2):279-290.

4. Picard C, et al. Primary Immunodeficiency Diseases: an Update on the Classification from the International Union of Immunological Societies Expert Committee for Primary Immunodeficiency 2015. J Clin Immunol. 2015;35(8):696-726.

5. Arkwright PD, Abinun M, Cant AJ. Autoimmunity in human primary immunodeficiency diseases. Blood. 2002;99(8):2694-2702.

6. Conley ME, Casanova JL. Discovery of single-gene inborn errors of immunity by next generation sequencing. Curr Opin Immunol. 2014;30:17-23.

7. de Beaucoudrey L, et al. Revisiting human IL-12R $\beta 1$ deficiency: a survey of 141 patients from 30 countries. Medicine (Baltimore). 2010;89(6):381-402.

8. Kuehn HS, et al. Immune dysregulation in human subjects with heterozygous germline mutations in CTLA4. Science. 2014;345(6204):1623-1627.

9. Marson A, Housley WJ, Hafler DA. Genetic basis of autoimmunity. J Clin Invest. 2015;125(6):2234-2241.

10. Massaad MJ, et al. Deficiency of base excision repair enzyme NEIL3 drives increased predisposition to autoimmunity. JClin Invest. 2016;126(11):4219-4236

11. Lee-Kirsch MA, Wolf C, Gunther C. Aicardi- 
Goutieres syndrome: a model disease for systemic autoimmunity. Clin Exp Immunol. 2014;175(1):17-24.

12. Lopez-Herrera G, et al. Deleterious mutations in LRBA are associated with a syndrome of immune deficiency and autoimmunity. Am J Hum Genet. 2012;90(6):986-1001.

13. Alangari A, et al. LPS-responsive beige-like anchor (LRBA) gene mutation in a family with inflammatory bowel disease and combined immunodeficiency. J Allergy Clin Immunol. 2012;130(2):481-488.

14. Charbonnier LM, et al. Regulatory T-cell deficiency and immune dysregulation, polyendocrinopathy, enteropathy, X-linked-like disorder caused by loss-of-function mutations in LRBA. JAllergy Clin Immunol. 2015;135(1):217-227.

15. Revel-Vilk S, et al. Autoimmune lymphoprolifera- tive syndrome-like disease in patients with LRBA mutation. Clin Immunol. 2015;159(1):84-92.

16. Alkhairy OK, et al. Spectrum of phenotypes associated with mutations in LRBA. J Clin Immunol. 2016;36(1):33-45.

17. Gamez-Diaz L, et al. The extended phenotype of LPS-responsive beige-like anchor protein (LRBA) deficiency. J Allergy Clin Immunol. 2016;137(1):223-230. 